\title{
Adaptive Optical Transceivers: Concepts and Challenges
}

\author{
Darli A. A. Mello, Valery N. Rozental, Tiago C. Lima, Fernando C. Pereira, A. N. Barreto, \\ Marco Camera and Gianmarco Bruno
}

\begin{abstract}
Coherent detection and digital signal processing techniques have driven a remarkable development in optical transport technologies, enabling channels at $100 \mathrm{~Gb} / \mathrm{s}$ to be transmitted over thousands of kilometers. Future optical communications systems will achieve even higher data rates $(>400$ Gb/s) through the deployment of superchannels, a designation for subcarrier multiplexing in the optical domain. In addition, a software-defined configuration of modulation format, transmission rate and coding scheme will enable advanced features such as automatic bandwidth provisioning and optimized spectrum allocation. However, compared with the wireless environment, optical systems are still very primitive in terms of intelligence, because their installation and operation require highly skilled manpower. The solution to this problem are adaptive optical transceivers, able to sense the channel conditions and to adapt their operation parameters to extend reach and reduce power consumption. In this paper we review a set of enabling concepts and algorithms of an adaptive optical transceiver, and discuss the challenges for its successful implementation.
\end{abstract}

Index Terms-Optical Communications, Coherent Detection, Adaptive Optical Transmission.

\section{INTRODUCTION}

Optical communications systems are undergoing a major transmission capacity upgrade, achieving data rates beyond $100 \mathrm{~Gb} / \mathrm{s}$ per wavelength with the combination of coherent detection, multilevel modulation formats and polarization multiplexing. At $400 \mathrm{~Gb} / \mathrm{s}$ and $1 \mathrm{~Tb} / \mathrm{s}$, bandwidth limitations imposed by analog-to-digital converters (ADCs) require the use of sub-carrier multiplexing, which in optical communications has been called superchannel technology [1]. The optical network is also being prepared for such upgrades with the deployment of a flexible grid, where the $50-\mathrm{GHz}$ ITU-T grid is replaced by a fine-granularity channelization plan that allows to set up channels with different bandwidths [2]. As transmission and networking technologies become more flexible, there is an increasing pressure for optimized network operation in terms of cost and power consumption [3]. Therefore, to improve network efficiency, future optical networks should bear adaptive properties, being able to selfadjust to changing transmission and traffic conditions [4].

In the physical layer of future networks, the key component is an adaptive optical transceiver, as depicted in Fig. 1. Performance requirements such as the net bit rate and required

D. A. A. Mello is with the Department of Communications (DECOM) of the School of Electrical and Computer Engineering (FEEC), University of Campinas, Brazil. V. N. Rozental, T. C. de Lima, F. C. Pereira and A. N. Barreto are with the OCNLab of the University of Brasília, Brazil. Marco Camera and Gianmarco Bruno are with Ericsson Telecomunicazioni, Genova. This work was supported by the Innovation Center, Ericsson Telecomunicações S.A., Brazil.

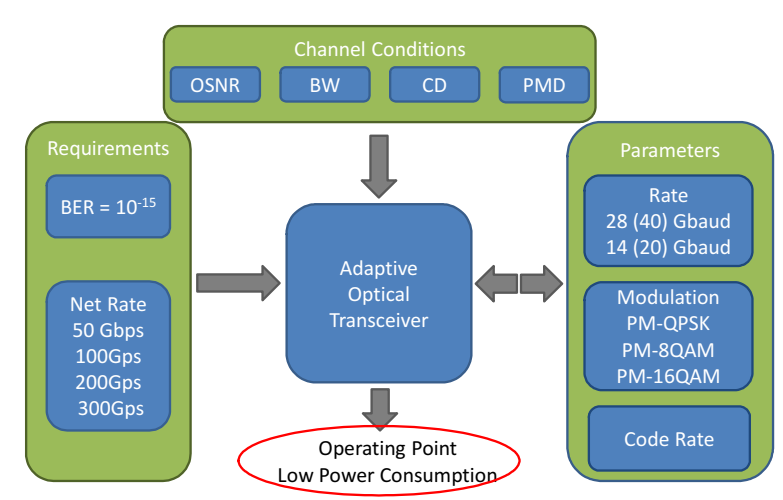

Fig. 1. Architecture of an adaptive transceiver.

bit error rate (BER) are provided by the control plane (left box). The transceiver senses the channel (upper box) to select an operating point (right box), which, fulfilling the system requirements, optimizes some cost function, usually power consumption. We denote an operating point as being the set of parameters that define the transceiver performance, in particular, symbol rate, modulation format, chain of signal processing algorithms and coding scheme.

Online channel identification is a crucial feature of future adaptive transponders. Given the widespread adoption of coherent detection and DSP techniques in optical systems, it is very likely that channel sensing will be based on the information provided by DSP algorithms, avoiding the use of additional optical performance monitoring hardware [5]. Therefore, in this paper, we consider DSP-based channel estimation techniques only. The main degradations in a high speed optical network are amplified spontaneous-emission noise (ASE), chromatic dispersion (CD), polarization-mode dispersion (PMD), narrowband filtering (BW), and nonlinearities. In long-haul transmission, $\mathrm{CD}$ has a stable and long impulse response, in the order of the duration of hundreds of symbols. It is compensated for by static digital filters whose coefficients need to be estimated by the receiver during the system startup. On the other hand, PMD has a dynamic and relatively short impulse response (usually a fraction of a symbol duration, reaching some few symbols in some cases), and is seamlessly compensated for in an adaptive butterfly filter with a small number of taps [6], typically below 10. Therefore, in general, PMD does not require estimation. Narrowband filtering is generated by the combined effect of reconfigurable add-drop multiplexers (ROADMs) and possible legacy dispersion compensation modules along the optical 
path. The frequency response of these components is known, change little over time, and could be used by the control plane to select a suitable lightpath. Narrowband filtering has not deserved specific compensating modules in current receiver proposals, except for some recent papers exploring signal predistortion [7]. It is interesting to observe that part of the degradations generated by narrowband filtering are also compensated for at the adaptive butterfly filter. Hence, the main effects to be estimated are ASE, nonlinearities, and CD.

This paper reviews a set of enabling concepts and algorithms of an adaptive optical transceiver, and discusses the challenges for its successful implementation. Section II reviews works on digital signal processing techniques for channel estimation. Section III examines the variables involved in parameter selection in regenerated and unregenerated optical systems. Lastly, Section IV concludes the paper.

\section{Channel Sensing}

\section{A. ASE Noise and Nonlinearities}

The signal degradation caused by ASE noise is commonly quantified by the optical signal-to-noise ratio:

$$
\mathrm{OSNR}_{\mathrm{ASE}}=\frac{P_{\mathrm{TX}}}{P_{\mathrm{ASE}}},
$$

where $P_{\mathrm{TX}}$ is the signal optical power, and $P_{\mathrm{ASE}}$ the optical noise power (usually modelled as additive white Gaussian

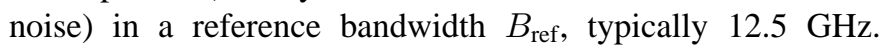
Under high power the optical fiber exhibits a nonlinear behavior, mainly caused by a nonzero third-order nonlinear susceptibility, known as Kerr effect. It was recently shown that, in dispersion-uncompensated optical links, nonlinearities are also Gaussian distributed [8]. In this way, it is convenient to define an equivalent optical signal-to-noise ratio, OSNR, including both ASE-noise and nonlinear interference (NLI) [9] contributions. Assuming unsaturated operation for optical amplifiers:

$$
\text { OSNR }=\frac{P_{\mathrm{TX}}}{P_{\mathrm{ASE}}+P_{\mathrm{NLI}}} .
$$

The art of optical systems design consists in finding the optimum fiber launch power that maximizes OSNR for a given optical link. In general, increasing $P_{\mathrm{TX}}$ also enhances $P_{\mathrm{NLI}}$. Reducing $P_{\mathrm{TX}}$ alleviates $P_{\mathrm{NLI}}$, but $P_{\mathrm{ASE}}$, which is independent on the signal launch power, dominates the system performance. Interestingly, recent results have also shown that, at the optimum fiber launch power, $P_{\mathrm{NLI}}=P_{\mathrm{ASE}} / 2$, i.e., the ASE noise power is twice that of nonlinear interference. Thus, the ratio between $P_{\mathrm{NLI}}$ and $P_{\mathrm{ASE}}$ is a helpful information for the system designer. For example, $P_{\mathrm{NLI}} / P_{\mathrm{ASE}}>0.5$ would be an indication of excessive signal launch power.

ASE noise and nonlinear interference may be distinguished by a slight correlation between the amplitude of neighboring symbols [10]. Let $r_{k}$ be the $\mathrm{k}$-th received symbol after equalization and phase recovery; $\hat{s}_{k}$ the decided symbol; and $\Delta_{k}=\left|r_{k}\right|-\left|\hat{s}_{k}\right|$. Assuming a stationary process, the autocorrelation function across neighboring $\Delta_{k}$ values is given by:

$$
R_{\Delta}(m)=\mathbb{E}\left\{\Delta_{k} \Delta_{k+m}\right\}
$$

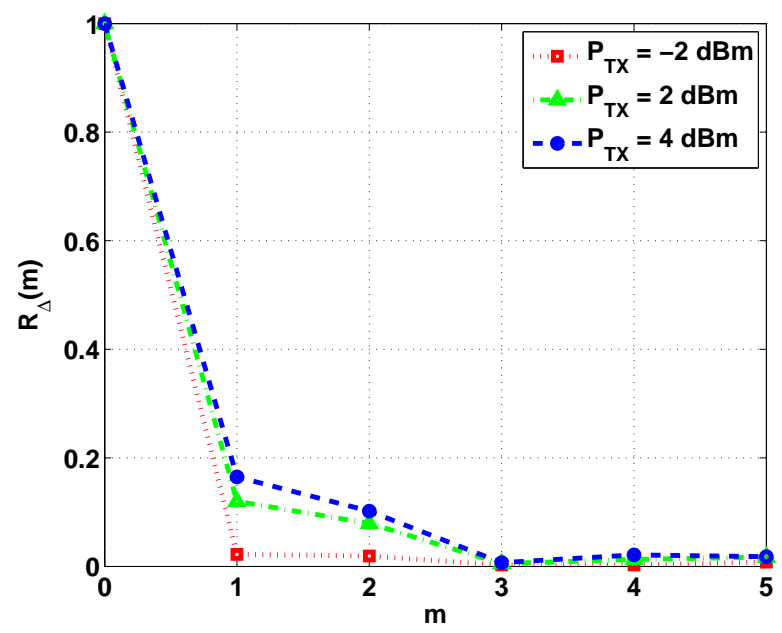

Fig. 2. Autocorrelation function across neighboring symbols.

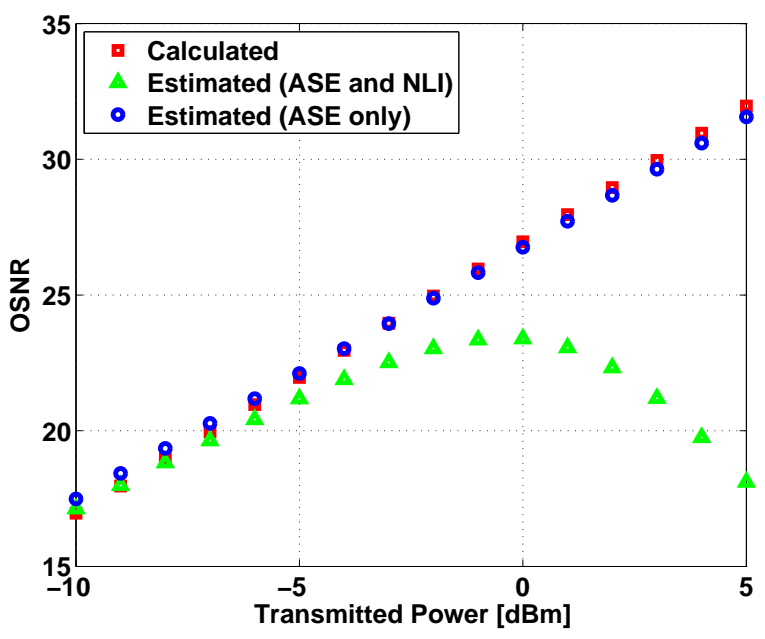

Fig. 3. Estimated OSNR by the technique in [11] for different scenarios.

where $\mathbb{E}\{\cdot\}$ denotes the expected value. Fig. 2 shows simulated $\left|R_{\Delta}\right|$ for a $100 \mathrm{G}$ QPSK signal with polarization multiplexing (PM-QPSK) generated by the Photoss software package. The simulated link consisted of 10 spans of $80-\mathrm{km}$ SSMFs and amplifiers with $5-\mathrm{dB}$ noise figure. The transmit power $\left(P_{\mathrm{TX}}\right)$ was varied from $-2 \mathrm{dBm}$ to $4 \mathrm{dBm}$, while the received power was kept at a constant value. Clearly, the autocorrelation for $m=1$ and $m=2$ increases with the transmit power due to nonlinearities. Indeed, Dong et al. [10] propose the use $R_{\Delta}$ to individually estimate $P_{\mathrm{ASE}}$ and $P_{\mathrm{NLI}}$, however, the estimation algorithm still requires a complex calibration process, which may be impractical in real-world optical systems. Estimating OSNR $_{\mathrm{ASE}}$ or $P_{\mathrm{NLI}} / P_{\mathrm{ASE}}$ in the nonlinear regime is still an open research topic, and one of the challenges of future adaptive optical transceivers.

In PM-QPSK systems, estimates of the optical signal-tonoise ratio, $\overline{\mathrm{OSNR}}$, have been been obtained by the technique presented by Ives et al. in [11]: 


$$
\begin{aligned}
\overline{\mathrm{SNR}} & =\frac{\sqrt{2 \mathbb{E}\left\{\left|r_{k}\right|^{2}\right\}^{2}-\mathbb{E}\left\{\left|r_{k}\right|^{4}\right\}}}{\mathbb{E}\left\{\left|r_{k}\right|^{2}\right\}-\sqrt{2 \mathbb{E}\left\{\left|r_{k}\right|^{2}\right\}^{2}-\mathbb{E}\left\{\left|r_{k}\right|^{4}\right\}}}, \\
\overline{\mathrm{OSNR}} & =\frac{p R_{s}}{2 B_{\mathrm{ref}}} \overline{\mathrm{SNR}}
\end{aligned}
$$

where $p$ is the number of polarization orientations used for signal transmission ( 1 for single-polarization systems and 2 for dual-polarization systems) and $R_{s}$ is the symbol rate. The bars over OSNR and SNR indicate estimated values. While OSNR was defined in (2) as the ratio of optical signal power and optical noise power in a reference bandwith $B_{\text {ref }}$, the electrical SNR follows the standard definition in communications theory, as the ratio of the signal energy $E$ by the noise power spectral density $N_{0}$. For a detailed discussion about OSNR and SNR please see [12]. It is interesting to observe that (4) may also be applied before phase recovery, as the method requires amplitude information only.

Fig. 3 illustrates the problem of distinguishing between $P_{\mathrm{NLI}}$ and $P_{\mathrm{ASE}}$ in the presence of nonlinearities. It shows $\overline{\mathrm{OSNR}}$ for a 100G PM-QPSK signal operating at both the linear (blue circles) and nonlinear (green triangles) regimes. The curves were obtained by Monte-Carlo simulation using the Photoss simulation package (again, 10 spans of $80-\mathrm{km}$ SSMFs and amplifiers with 5-dB noise figure). The analytical OSNR (red squares) was computed by the traditional link power budget equation [13, p. 174] for optical links with saturated amplifiers and constant amplifier spacing:

$$
\begin{aligned}
\operatorname{OSNR}_{\mathrm{ASE}}=P_{\mathrm{TX}}-\mathrm{F}-10 \cdot \log _{10}\left(N_{s}\right) & \\
& -\alpha_{\mathrm{dB} / \mathrm{km}} \cdot L_{s}-10 \cdot \log _{10}\left(h \nu B_{\mathrm{ref}}\right),
\end{aligned}
$$

where $\mathrm{F}$ is the EDFA noise figure, $N_{s}$ is the number of spans, $\alpha_{\mathrm{dB} / \mathrm{km}}$ the fiber loss coefficient, $L_{s}$ the span length, $h$ the Planck constant, and $\nu$ the carrier frequency.

When the transmit power is low (that is, nonlinear effects are negligible), the three curves coincide. At high launch powers, if the nonlinear channel model is adopted, $\overline{\text { OSNR }}$ decreases as expected, because the estimaton algorithm is unable to discriminate between linear and nonlinear noises.

\section{B. CD Estimation}

Legacy optical systems manage chromatic dispersion with Bragg-grating-based dispersion compensation modules (DCMs) or dispersion-compensating fibers (DCFs). However, current and future coherent optical systems will rely on electronic dispersion compensation techniques, avoiding optical compensation. There are two main advantages for this choice: first, from a management perspective, it is opportune to replace hundreds of DCMs or DCFs by a simple DSP element embedded into the receiver; second, removing dispersion compensation modules eliminates the associated insertion loss, resulting in a higher OSNR at the end of the link.

Adaptive transceivers need to accurately estimate $\mathrm{CD}$ to be seamlessly integrated into links of different lengths and fiber types. Several algorithms have been proposed for CD estimation. Most of them use scanning techniques that apply

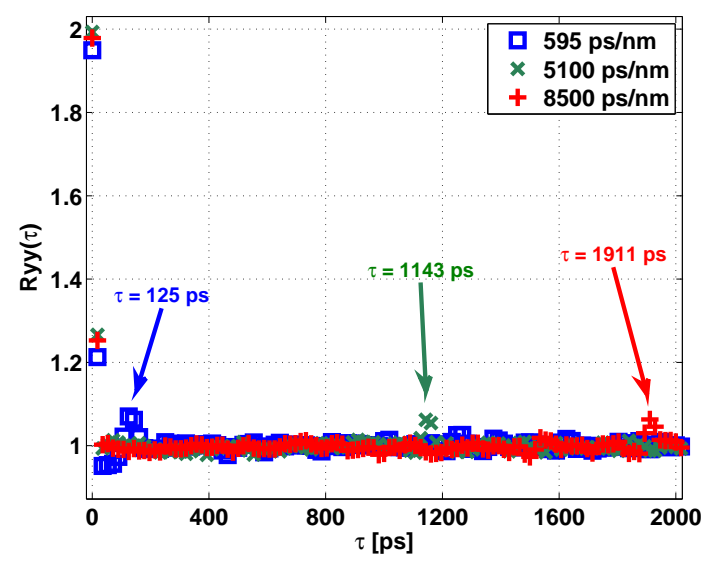

Fig. 4. Simulated autocorrelation functions for selected CD values.

the $\mathrm{CD}$ compensation filter to the received signal assuming some accumulated dispersion, and compute a certain cost function related to the signal quality. Channel dispersion is estimated as the one that minimizes the cost function. As an example, a technique devised by Kuschnerov et al. in [14] uses an error criterion based on Godard's constant modulus algorithm [15]. The method consists of two parts: first, the error is computed within a possible $\mathrm{CD}$ range in discrete steps of $200 \mathrm{ps} / \mathrm{nm}$. A CD value for which the error is at minimum is found. Then, the error is calculated at a higher resolution, in discrete steps of $20 \mathrm{ps} / \mathrm{nm}$ around the $\mathrm{CD}$ value found in the first part. The main advantage of this algorithm is simplicity, although it has to be executed for many possible CD values. In [16], Borkowski et al. validated four different scanning algorithms using a PM-QPSK setup.

Alternatively, an accurate and computationally efficient CD estimation technique (auto-correlation signal power waveform ACSPW) was proposed by Sui et al. in [17]. The paper demonstrates a relation between the position of the peak of the signal power autocorrelation and accumulated $\mathrm{CD}$. In mathematical terms, for high accumulated $\mathrm{CD}$, the signal power autocorrelation function, $R_{y y}(\tau)$, exhibits a peak at $\tau_{0}$. The accumulated $\mathrm{CD}$ is related to $\tau_{0}$ by [17]:

$$
\mathrm{CD}_{\mathrm{acc}}=\frac{\tau_{0} T_{s}+\sqrt{\tau_{0}^{2} T_{s}^{2}-16 \pi^{2} T_{0}^{4}}}{4 \pi} \frac{2 \pi c}{\lambda^{2}},
$$

where $T_{s}$ is the symbol period, $T_{0}$ is the Gaussian pulse halfwidth at the 1/e point, $c$ is the speed of light and $\lambda$ is the carrier wavelength. Fig. 4 depicts simulated autocorrelation functions for three different $C D$ values of 525,5100 and $8500 \mathrm{ps} / \mathrm{nm}$, obtained by the Photoss simulation software. The peaks appear in 125,1143 and $1911 \mathrm{ps}$, and, using these $\tau_{0}$ values in (7), the estimated CD is 543,5072 and $8482 \mathrm{ps} / \mathrm{nm}$, respectively.

It is important to note that (7) only gives positive results. Furthermore, in order for $\mathrm{CD}_{\text {acc }}$ to be real, the square root argument must be positive. This imposes a fundamental limitation to the ACSPW algorithm, that is, the algorithm requires a minimum accumulated dispersion to work properly. This may be a problem in dispersion managed links with little, or even negative, residual chromatic dispersion. Pereira et al. 


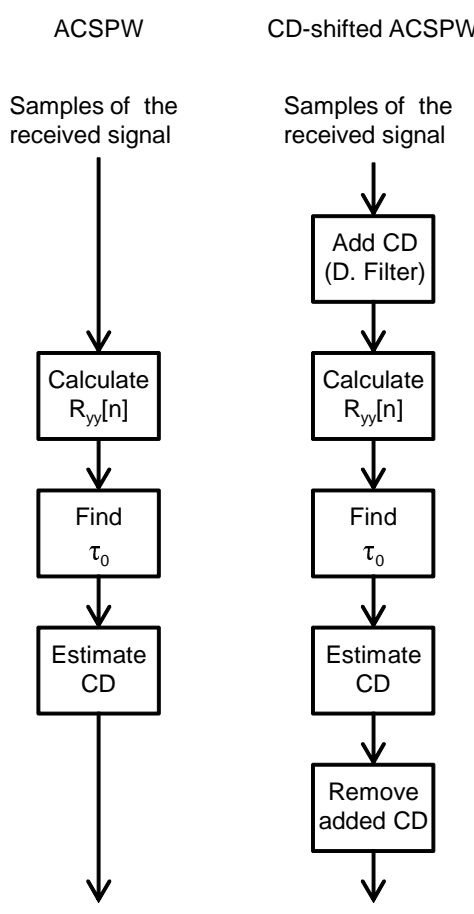

Fig. 5. Block diagram of ACSPW and CD-shifted ACSPW.

overcame this problem in [18] by the so-called CD-shifted ACSPW technique (see the block diagrams in Fig. 5). The idea is to use a digital filter to artificially add a fixed amount of $\mathrm{CD}$, guaranteeing that the accumulated $\mathrm{CD}$ is always higher than the minimum required. After estimation, which is made exactly as in ACSPW, the inserted CD value is subtracted from the estimated value.

\section{PARAmeter SElection}

Once the channel is estimated, the transmission parameters, mainly data rate, modulation scheme and DSP algorithms, must be set or updated accordingly. In the context of adaptive communication systems, data rates of $400 \mathrm{~Gb} / \mathrm{s}$ and $1 \mathrm{~Tb} / \mathrm{s}$ per optical channel must be regarded. However, while research on $100 \mathrm{~Gb} / \mathrm{s}$ systems has reached sufficient maturity and the first commercial transport interfaces have been deployed, higher data rates are still challenging. The main limitations are the bandwidths of optical and electrical components, and the resolution of ADCs. State-of-the-art ADCs operate at up to $60 \mathrm{GS} / \mathrm{s}$, limiting the bandwidth of detected channels [23]. Solutions that allow higher spectral efficiencies involve superchannels, tightly spaced optical subcarriers that are transmitted through the optical network as a single entity [1][24], but detected independently (see Table I for examples of Terabit superchannels). Superchannels usually have $1 / T_{s}$ or a slightly higher frequency spacing between subcarriers, where $T_{s}$ is the symbol duration. These solutions attempt to achieve the highest possible subcarrier bitrates that can still be handled electronically, while keeping optical parallelization to a minimum.

The next sections tackle the problem of parameter selection for a single channel,but the conclusions also apply to indi- vidual subcarriers of superchannels. The discussion is divided into two sections. Section III-A addresses long-haul optical links that do not require optical-electrical-optical regeneration. Section III-B concerns regenerated links. This is because the paradigms for finding the optimal operating point are different for the two cases. In unregenrated links the cost of the solution is given by the two transceivers in the end of the link, and the best operating point minimizes power consumption for a fixed reach. In regenerated links, however, the optimization process minimizes the number of extremely expensive regenerators (and, consequently, cost), by selecting the operating point with the longest transparent reach, irrespective of its the power consumption. Note that although the main objective is to minimize cost, regenerators are also power hungry, and the overall power consumption is also minimized.

\section{A. Unregenerated Links}

In unregenerated optical links, the optimum operating point minimizes power consumption while satisfying traffic and reach requirements. In the example of [25], the most power consuming components of an optical transceiver are the DSP (28\%), followed by the deframer (7\%) and drivers $(2.5 \%)$. Hence, power-efficient DSP algorithms can achieve a reasonable reduction in the transceiver power consumption and, for this reason, they are the focus of this section.

We based our study on the following assumptions:

- Fixed-rate forward error correction (FEC). We assume a $10^{-3}$ pre-FEC BER, required for a $10^{-15}$ post-FEC BER using $7 \%$ overhead, as in standard 100G PM-QPSK systems [26].

- A maximum symbol rate, $R_{s}^{\max }$, of 28 Gbaud. This rate is widely used $100 \mathrm{G}$ PM-QPSK long-haul transmission, allowing T/2-spaced sampling using commercially available ADCs [27].

- Discrete symbol rates of the form $R_{s}^{\max } / 2^{l}, \quad l=$ $0,1,2,3$, resulting in rates of $3.5,7,14$ and 28 Gbaud. These rates can be easily implemented by symbol repetition [28].

- Reach and accumulated CD independence. We assume the general case where the optical route may contain legacy CD compensation modules. In this scenario, accumulated $\mathrm{CD}$ is not necessarily a function of the optical link length.

Future software-defined optical transceivers will have the ability to choose the modulation format according to the channel conditions [29]. In this study, we assume the most simple digital modulation formats: BPSK, QPSK, 8QAM and 16QAM. In fixed-code rate transceivers, higher-order modulation formats would have a prohibitively high OSNR penalty.

\section{Required OSNR (OSNR req$)$}

The combinations of modulation format (BPSK, QPSK, 8QAM or 16QAM) and symbol rate $(3.5,7,14$ or 28 Gbaud) achieve several transmission rates: $12.5,25,37.5,50,75,100$, 150 and $200 \mathrm{~Gb} / \mathrm{s}$. Each of these rates (except 150 and 200 $\mathrm{Gb} / \mathrm{s}$ ) have more than one possible form of transmission. As an example, Table II summarizes combinations resulting in 
TABLE I

SUPERCHANNELS FOR TERABIT OPTICAL TRANSMISSION

\begin{tabular}{c|c|c|c|c|c|c} 
Aggregate Rate [Gb/s] & Modulation Format & [Bits/Symbol] & Subcarriers & Symbol Rate [GSymbols/s] & Ref. & Year \\
\hline \hline 1,284 & PM-64QAM & 12 & 10 & 10.7 & 50.25 & {$[19]$} \\
1,206 & PM-16QAM & 8 & 3 & 12.5 & 2012 \\
1,200 & PM-QPSK & 4 & 24 & 24.8 & 2013 \\
$1,582.2$ & PM-16QAM & 8 & 8 & {$[21]$} & 2013 \\
\hline
\end{tabular}

TABLE II

POSSIBLE COMBINATIONS FOR 50-GB/S TRANSMISSION

\begin{tabular}{c|l|c} 
Nominal Rate $[\mathrm{Gb} / \mathrm{s}]$ & Transmission Scheme & OSNR $_{\text {req }}[\mathrm{dB}]$ \\
\hline \hline \multirow{5}{*}{50} & PM-BPSK @ 28 Gbaud & 10.3 \\
& PM-QPSK @ 14 Gbaud & 10.3 \\
& SP-QPSK @ 28 Gbaud & 10.3 \\
& PM-16QAM@ 7 Gbaud & 14.0 \\
& SP-16QAM @14 Gbaud & 14.0 \\
\hline \hline
\end{tabular}

$50 \mathrm{~Gb} / \mathrm{s}$ transmission (PM stands for "polarization multiplexing", and SP for "single polarization"). Each transmission scheme has an associated required optical signal-to-noise ratio OSNR $_{\text {req }}$, as shown in the third column of Table II, which is directly related to reach.

The calculation of $\mathrm{OSNR}_{\text {req }}$ follows the steps below. Assuming coherent detection, Gray coding for QPSK and 16QAM, and optimal coding for 8QAM, BER and signal-to-noise ratio per bit $\mathrm{SNR}_{b}{ }^{1}$ are related by [30]:

$$
\begin{aligned}
\operatorname{BER}(\mathrm{BPSK}, \mathrm{QPSK}) & =\frac{1}{2} \operatorname{erfc}\left(\sqrt{\mathrm{SNR}_{b}}\right) \\
\operatorname{BER}(8 \mathrm{QAM}) & \approx \frac{11}{16} \operatorname{erfc}\left(\sqrt{\frac{3 \mathrm{SNR}_{b}}{3+\sqrt{3}}}\right) \\
\operatorname{BER}(16 \mathrm{QAM}) & \approx \frac{3}{8} \operatorname{erfc}\left(\sqrt{\frac{6 \mathrm{SNR}_{b}}{15}}\right),
\end{aligned}
$$

where erfc is the complimentary error function. For the target $\mathrm{BER}=10^{-3}$, Eqs. 8 to 10 yield [30]:

$$
\begin{aligned}
\mathrm{SNR}_{b}(\mathrm{BPSK}, \mathrm{QPSK}) & =6.8 \mathrm{~dB} \\
\mathrm{SNR}_{b}(8 \mathrm{QAM}) & =9.0 \mathrm{~dB} \\
\mathrm{SNR}_{b}(16 \mathrm{QAM}) & =10.5 \mathrm{~dB} .
\end{aligned}
$$

Finally, $\mathrm{OSNR}_{\text {req }}$ is obtained from $\mathrm{SNR}_{b}$ by [12]:

$$
\mathrm{OSNR}_{\text {req }}=\frac{R_{b}}{2 B_{\text {ref }}} \mathrm{SNR}_{b},
$$

where $R_{b}$ is the transmission bit rate. Note in Table II that, for the same transmission rate, BPSK and QPSK signals require the same OSNR, and so do single- and dual-polarization signals.

\section{Computational Complexity Analysis}

In dispersion-uncompensated optical transmission, long CD compensation filters (with potentially hundreds of taps) are the

\footnotetext{
${ }^{1} \mathrm{SNR}_{b}=\mathrm{SNR} / \tilde{R}$, where $\tilde{R}$ is the number of information bits conveyed per modulation symbol.
}

primary contribution for power consumption and digital footprint in the DSP module. The length of the CD compensation bulk filter, $N_{\mathrm{CD}}$, can be computed by [31]:

$$
N_{\mathrm{CD}}=2 \times\left\lfloor\frac{\mathrm{CD}_{\mathrm{acc}} \lambda^{2}}{2 c T_{\mathrm{sp}}^{2}}\right\rfloor+1,
$$

where $\mathrm{CD}_{\mathrm{acc}}$ is the accumulated $\mathrm{CD}, \lambda$ is the optical carrier wavelength, $T_{s p}$ is the sampling time and $c$ is the speed of light. Note that the filter size is a linear function of $\mathrm{CD}_{\mathrm{acc}}$, and a quadratic function of the symbol rate (for T/N-spaced filters, $R_{s}=1 /\left(N T_{s p}\right)$ ), independently of the modulation format. This feature plays an important role in assessing the efficiency of the distinct modulation formats.

After obtaining the filter size, it is possible to estimate the computational complexity associated with CD compensation. Our estimation is based on the number of real multiplications, which is the dominating parameter [32]. We focus on frequency-domain equalization (FDE), because of its computational efficiency in comparison with time-domain equalization for a sufficient filter length [28][33]. The FDE filtering cycle involves the following operations:

1) Computation of the fast Fourier transform (FFT) of the filter coefficients (this operation is performed only once and does not contribute to the complexity.)

2) Calculation of the FFT of a new-coming data-block.

3) Term-by-term multiplication of the results of steps (1) and (2).

4) Computation of the inverse FFT of the result.

The number of (non-trivial) real multiplications, $M_{R}$, required for the computation of an FFT of size $N_{\text {FFT }}$, depends on the algorithm, optimization degree and hardware implementation. Here, we assume a radix-2 Cooley-Tukey algorithm, because it can be implemented for any power of two FFT size, yielding the desired flexibility for rate-adaptive schemes. We further assume an optimized FFT, where the implementation of a complex multiplication corresponds to three real ones. ${ }^{2}$ Under these conditions, $M_{R}$ is given by [34]:

$$
M_{R}=\frac{3}{2} N_{\mathrm{FFT}}\left(-3+\log _{2} N_{\mathrm{FFT}}\right)+6,
$$

provided that $N_{\mathrm{FFT}}=2^{k}, k \in \mathbb{N}$.

The number of real multiplications per filtering cycle, $M_{\mathrm{CYC}}$, is given by:

$$
M_{\mathrm{CYC}}=p\left(2 M_{R}+3 N_{\mathrm{FFT}}\right)
$$

${ }^{2}(\mathrm{e}+\mathrm{jf})=(\mathrm{a}+\mathrm{jb}) \cdot(\mathrm{c}+\mathrm{jd})$ can be computed as $\mathrm{e}=(\mathrm{a}-\mathrm{b}) \mathrm{d}+\mathrm{a}(\mathrm{c}-\mathrm{d})$ and $\mathrm{f}=(\mathrm{a}-$ $b) d+b(c+d)$. This form requires three real multiplications and five real additions [34]. 


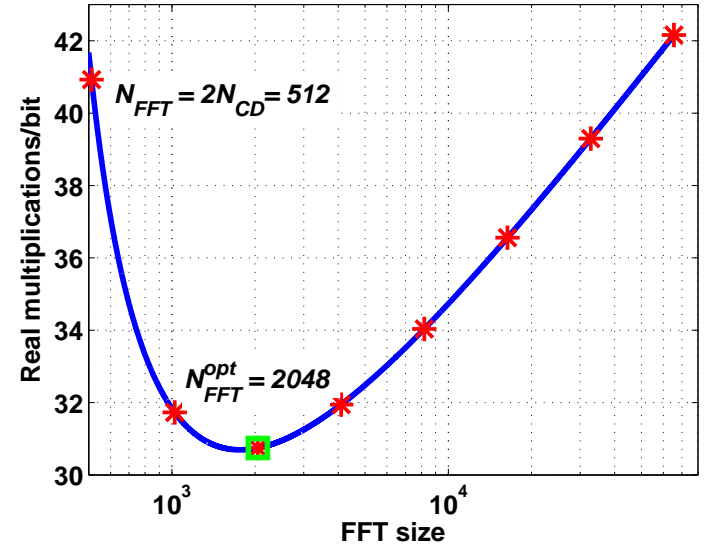

Fig. 6. FFT size optimization for a 256 taps CD compensation filter.

where $p$ is the number of polarizations. In (17), $M_{R}$ is multiplied by 2 to account for both the FFT and the subsequent IFFT. The $N_{\text {FFT }}$ parameter is multiplied by 3 , as three real multiplications are required for each complex one.

The number of bits resulting in one filtering cycle, $b_{\mathrm{CYC}}$, is given by:

$$
b_{\mathrm{CYC}}=\frac{p L}{\kappa} \log _{2} \mathrm{M}
$$

where $L$ is the number of equalized samples resulting from one filtering cycle in one polarization, $\kappa$ is the oversampling factor and $\mathrm{M}$ is the size of the modulation alphabet. Finally, the number of multiplications per transmitted bit, $M_{b}$, is computed as:

$$
\begin{aligned}
M_{b} & =M_{\mathrm{CYC}} / b_{\mathrm{CYC}} \\
& =\frac{\kappa}{L \log _{2} \mathrm{M}}\left(2 M_{R}+3 N_{\mathrm{FFT}}\right) .
\end{aligned}
$$

Note that $M_{b}$ does not depend on the number of polarization orientations.

Frequency-domain equalization of long data sequences requires an overlapping method. In this way, the number of equalized samples resulting from one filtering cycle, $L$, in (19), is related to the FFT size, $N_{\mathrm{FFT}}$, by [35]:

$$
L=N_{\mathrm{FFT}}-N_{\mathrm{CD}}+1 \text {. }
$$

Obtaining aliasing-free equalization requires a minimum of $50 \%$ overlap, that is, an FFT size of at least twice the filter length [35]. However, $M_{b}$, in (19), can be minimized with respect to $N_{\mathrm{FFT}}$, significantly reducing the complexity [36]. Fig. 6 illustrates this procedure by showing the number of multiplications per transmitted bit for a 256 tap CD compensation filter for a PM-16QAM signal, as a function of the FFT size. This is equivalent to the size of a $T / 2$-spaced equalizer for a 14 Gbaud sinal transmitted over nearly $2400 \mathrm{~km}$ of SSMF, calculated by (15). Here, the red stars represent possible $N_{\text {FFT }}$ values, and the green square indicates the optimum FFT size, $N_{\mathrm{FFT}}^{\mathrm{opt}}$. In this case, the FFT size optimization resulted in roughly $25 \%$ complexity reduction in comparison with the $50 \%$ overlap case. The optimum FFT size values can be precomputed and stored in hardware. Fig. 7 shows the optimum

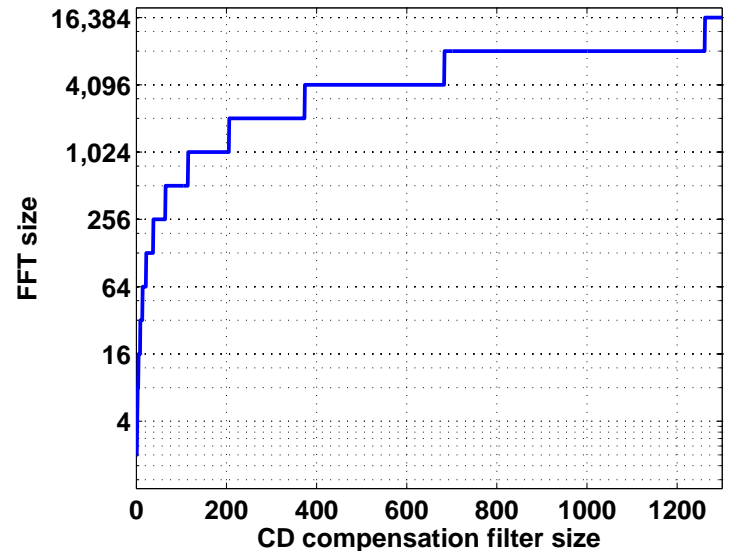

Fig. 7. Optimal FFT size values.

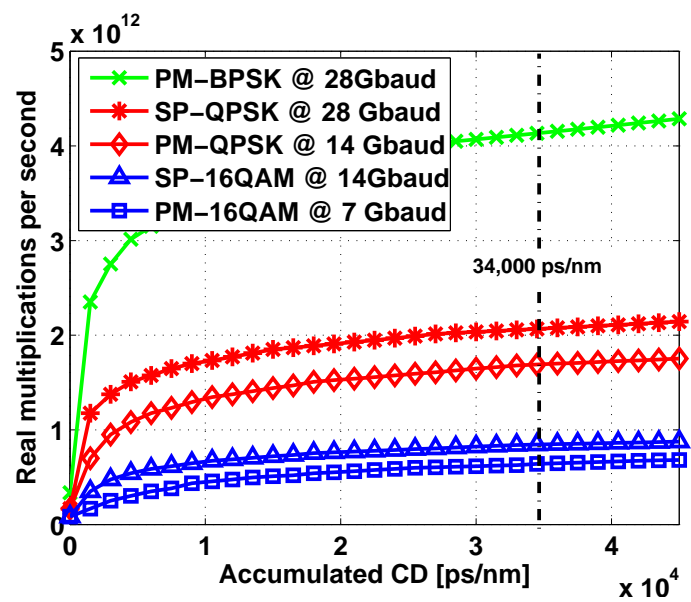

Fig. 8. Computational complexity of the $50 \mathrm{~Gb} / \mathrm{s}$ transmission schemes.

FFT size values for different filter lengths and a wide range of practical accumulated $\mathrm{CD}$ values.

\section{Pareto-Efficiency Analysis}

We next analyze the suitability of an operating point through the combination of two parameters: computational complexity for a given accumulated CD value, and ROSNR for a given BER. The former is directly related to power consumption, and the latter to reach. Fig. 8 displays the computational complexity of the transmission schemes of Table II as a function of accumulated CD. Note that the relative distance between the curves remains quasi-constant throughout a wide range of high accumulated CD values. This allows us to simplify the analysis assuming some (sufficiently high) fixed value of accumulated $\mathrm{CD}$, without loss of generality. From the inspection of Table II and Fig. 8, it is evident that neither single-polarization schemes nor BPSK are advantageous compared to other options using polarization-multiplexed transmission with I/Q modulation.

This can be viewed in terms of Pareto efficiency over ROSNR and computational complexity. Given a set of possible operating points, a change to an operating point that improves one parameter (ROSNR or computational complexity) without 


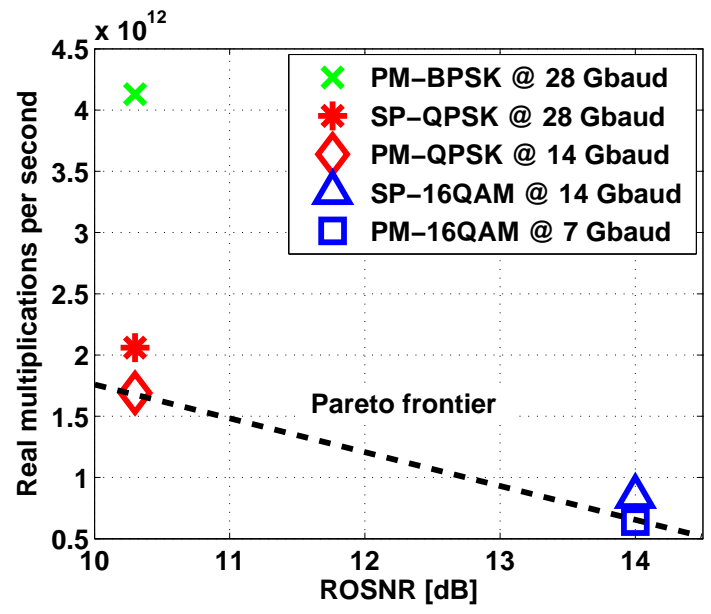

Fig. 9. Pareto efficiency for $50 \mathrm{~Gb} / \mathrm{s}$ transmission schemes.

making others worse is called a Pareto improvement. An operating point is denoted as Pareto efficient when no further Pareto improvements can be made from that point. The set of all Pareto-efficient operating points is called a Pareto frontier. Single-polarization schemes require a higher computational complexity than polarization-multiplexed schemes to achieve the same transmission rate, at the same ROSNR. Therefore, single-polarization schemes are never Pareto efficient. The comparison between QPSK and BPSK is analogous (QPSK is always preferable). Naturally, employing all available transmission dimensions (polarization multiplexing, I/Q transmission) reduces the system symbol rate and, as a consequence, the computational complexity.

As an illustrative example, consider a fixed value for accumulated CD, 34,000 ps/nm (black dash-dotted line in Fig. 8), corresponding to $2000 \mathrm{~km}$ of standard single-mode fiber (SSMF) with dispersion coefficient $D=17 \mathrm{ps} / \mathrm{nm} / \mathrm{km}$. Potential transmission schemes may be arranged as in Fig. 9. Here, the Pareto frontier is formed by PM-QPSK and PM16QAM. Other schemes do not meet the Pareto-efficiency criterion, i.e., are not beneficial neither in ROSNR nor in power consumption, and should not be employed.

An analogous analysis for all considered transmission rates results in the set of Pareto-efficient schemes summarized in Table III. The complexity values shown in the fourth column correspond to the transmission over $2000 \mathrm{~km}$ SSMF. The table also includes the complexity normalized with respect to its maximum (3.68 $\left.\mathrm{Tops}^{3}\right)$.

\section{B. Links with Optical-Electrical-Optical (OEO) Regeneration}

Long optical links require optical-electrical-optical (OEO) regeneration to recover the transmitted signal, an extremely expensive procedure which should be avoided whenever possible. Thus, in very long data links, from a cost perspective, the optimum operating point minimizes the number of regenerators. In the optical domain, this means to choose the set of parameters that maximizes reach. The maximum distance between regenerators, usually called transparent reach, is computed as

\footnotetext{
${ }^{3}$ Tera operations per second
}

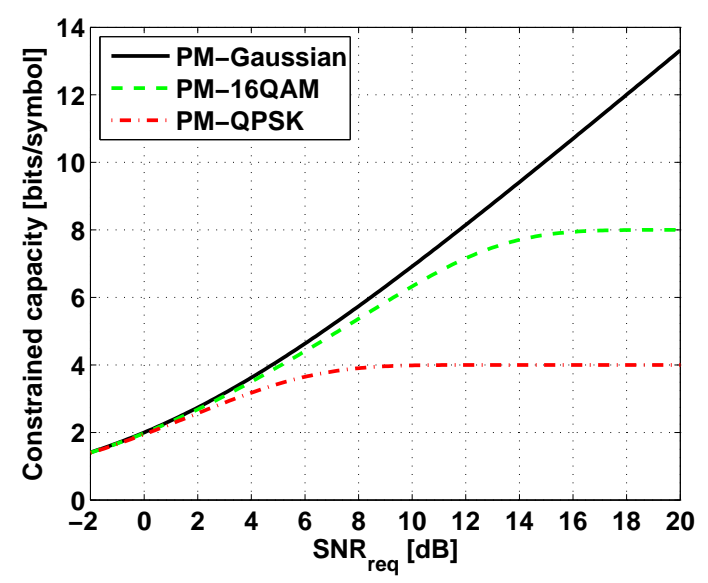

Fig. 10. Constrained capacity for PM-QPSK, PM-16QAM and PM-Gaussian formats.

the maximum length that satisfies OSNR $>$ ROSNR, where OSNR is the optical signal-to-noise ratio at the end of the link (as defined in (2)), and ROSNR is the minimum tolerable OSNR that results in an acceptable bit error rate (BER). In the dimensioning of practical systems, it is also common to add $3 \mathrm{~dB}$ to ROSNR as a safety margin to account for eventual aging and the degradation of system components.

\section{Modulation Format}

We start by selecting the modulation format which corresponds to the lowest ROSNR. In order to convey a client traffic demand of $R_{b}$ bits per second using a transmission device operating at $R_{s}$ symbols per second, a mutual information of $I=R_{b} / R_{s}$ bits per symbol is required. The maximum mutual information attained by a modulation format depends on the signal-to-noise ratio of the link, and is called constrained capacity. Fig. 10 shows the constrained capacity curves for the QPSK and 16QAM modulation formats, and the Shannon Limit, achieved if the input signal has a bidimensional Gaussian distribution [37, p. 242]. A detailed description of the constrained capacity calculation method can be found in [38]. All curves were evaluated considering polarization multiplexing. Once a modulation format is chosen, the electrical SNR required to achieve a certain mutual information, RSNR, can be obtained from Fig. 10. ROSNR, in turn, can be computed from RSNR using (5).

For example, let us suppose that the transceiver operates at a symbol rate of 28 Gbaud (as most commercial $100 \mathrm{~Gb} / \mathrm{s}$ systems), and that client traffic requires $100 \mathrm{~Gb} / \mathrm{s}$ including overhead. In this case, a mutual information of $100 / 28=$ $3.57 \mathrm{bit} / \mathrm{symbol}$ is required. Fig. 10 indicates an $\mathrm{SNR}_{\text {req }}$ of approximately $4 \mathrm{~dB}$ for 16QAM, and $6 \mathrm{~dB}$ for QPSK. It is interesting to note that, in this portion of the curve, the difference in performance between the 16QAM modulation format and the Shannon Limit is negligible. From (5), it is possible to verify that ROSNR is directly proportional to RSNR. Therefore, high-order modulation formats (with lower code rates) require a lower ROSNR than low-order alternatives (with higher code rates). There are, of course, issues related 
TABLE III

PARETO-EFFICIENT SCHEMES

\begin{tabular}{c|c|c|r|r} 
Nominal Rate [Gb/s] & Transmission Schemes & ROSNR [dB] & Complexity [Tops] & Normalized complexity [\%] \\
\hline \hline 12.5 & PM-QPSK @ 3.5 Gbaud & 4.3 & 0.19 & 5.2 \\
\hline \multirow{2}{*}{25} & PM-QPSK @ 7 Gbaud & 7.3 & 0.57 & 15.5 \\
& PM-16QAM@ 3.5 Gbaud & 11.0 & 0.19 & 5.2 \\
\hline 37.5 & PM-8QAM @ 7 Gbaud & 11.3 & 0.57 & 15.5 \\
\hline \multirow{2}{*}{50} & PM-QPSK @ 14 Gbaud & 10.3 & 1.51 & 41.0 \\
& PM-16QAM@ 7 Gbaud & 14.0 & 0.57 & 15.5 \\
\hline 75 & PM-8QAM @ 14 Gbaud & 14.3 & 1.51 & 41.0 \\
\hline \multirow{2}{*}{100} & PM-QPSK @ 28 Gbaud & 13.3 & 3.68 & 100.0 \\
& PM-16QAM@ 14 Gbaud & 17.0 & 1.51 & 41.0 \\
\hline \multirow{2}{*}{150} & PM-8QAM @ 28 Gbaud & 17.3 & 3.68 & 100.0 \\
\hline 200 & PM-16QAM@ 28 Gbaud & 20.0 & 3.68 & 100.0 \\
\hline
\end{tabular}

to the implementation of high-order modulation formats (e.g. DAC/ADC resolution), however, among the modulation formats implementable by the available technology, high-order modulation formats are preferable.

This conclusion is in apparent contradiction with the numbers in Table III, where higher-order modulation formats require a higher ROSNR (although reducing power consumption through a lower symbol rate). In the previous section we addressed parameter optimization in links without regeneration, where the best operating point minimizes power consumption for a given reach (translated in terms of ROSNR) and traffic requirements. In this section, however, we maximize reach irrespectively of power consumption. In order to choose the most suitable operating parameters, we allow in this section the code rate of error-correcting schemes to vary freely, contrasting with previous section where the FEC overhead was set to $7 \%{ }^{4}$. For example, the mutual information of $3.57 \mathrm{bit} / \mathrm{symbol}$ is achieved by a code rate of 3.57/4 $=0.8925$ for PM-QPSK and $3.57 / 8=0.44625$ for PM-16QAM. Working with code rates as low as 0.44 of course increases the transceiver power consumption because the symbol rate is high and the net information rate low, but reach is considerably extended and, as a result, regeneration is avoided. The ability to self-adjust the FEC code rate is a timely research challenge for adaptive optical transceivers [38][39][40][41].

\section{Symbol Rate}

In addition to modulation format and code rate, symbol rate is another important parameter to be optimized. According to (5), increasing $R_{s}$ also increases ROSNR, which may lead to the (erroneous) conclusion that raising the symbol rate degrades the system performance. However, one should note that increasing $R_{s}$ also decreases the mutual information required to achieve the same information bit rate and, consequently, the RSNR (see Fig. 10). This is because a higher $R_{s}$, for a constant required information rate, raises the amount of redundant information used by error-correcting schemes. Hence, if $R_{s}$ increases, RSNR decreases at a higher rate. The combined effect can be seen in Fig. 11, which shows an example for a

${ }^{4}$ Overhead, $\mathrm{OH}$, and code rate, $\mathrm{R}$, are related by $R=1 /(1+\mathrm{OH})$ data rate of $100 \mathrm{~Gb} / \mathrm{s}$.

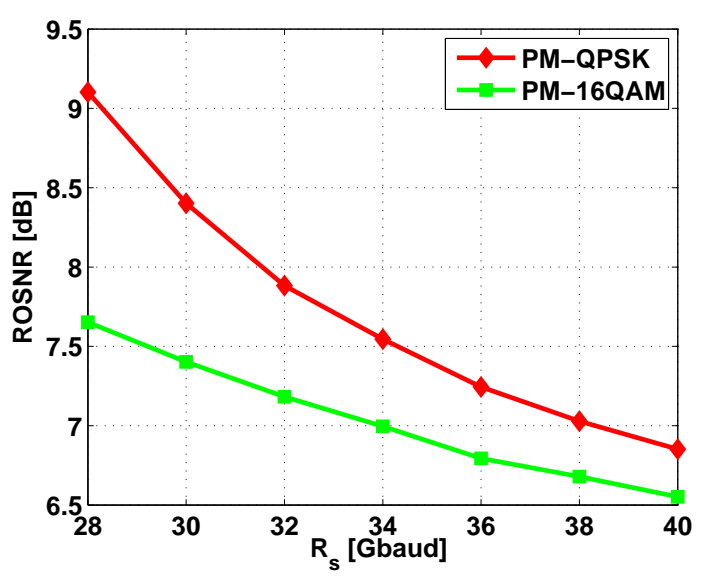

Fig. 11. Required OSNR versus symbol rate for $100 \mathrm{~Gb} / \mathrm{s}$ with PM-QPSK and PM-16QAM.

Another dimension influenced by the symbol rate is the power of nonlinear interference $P_{\mathrm{NLI}}$. To analyze the effect of the symbol rate on $P_{\mathrm{NLI}}$ we use the GN-model presented in $[42]^{5}$. The model computes the link OSNR according to (2), i.e., noise power is calculated as the sum of ASE noise power $P_{\mathrm{ASE}}$ and nonlinear interference power $P_{\mathrm{NLI}}$. $P_{\mathrm{ASE}}$ is evaluated by the standard formula for noise accumulation ${ }^{6}[13$, p. 173]:

$$
P_{\mathrm{ASE}}=N_{\mathrm{s}} F\left(e^{2 \alpha_{\mathrm{np} / \mathrm{km}} L_{s}}-1\right) h \nu B_{\mathrm{ref}} .
$$

Note that (6) can be obtained from (21) assuming saturated optical amplifiers, as in [13, pp. 173-174]. The nonlinear power spectral density $G_{\mathrm{NLI}}(f)$ can be assumed as locally flat within the reference bandwidth [45][8][42]. Thus, the nonlinear power per span, $P_{\mathrm{NLI}, \mathrm{SS}}$, within the reference bandwidth, $B_{\text {ref }}$, is given by:

$$
P_{\mathrm{NLI}, \mathrm{SS}}=G_{\mathrm{NLI}, \mathrm{SS}}(0) B_{\mathrm{ref}},
$$

\footnotetext{
${ }^{5}$ Analytical modeling of nonlinear propagation is currently subject to an intense debate in the scientific community. More details can be found in [43][44].

${ }^{6} 1 \alpha_{\mathrm{dB} / \mathrm{km}}=8.68 \alpha_{\mathrm{np} / \mathrm{km}}$.
} 
where $G_{\mathrm{NLI}, \mathrm{SS}}(0)$ is the nonlinear power spectral density at the center of the channel $(f=0)$ for a single span.

From the nonlinear interference model (derived in details in [42]), it can be shown that

$$
P_{\mathrm{NLI}, \mathrm{SS}}=\eta_{\mathrm{SS}} P_{\mathrm{TX}}^{3}
$$

where $P_{\mathrm{TX}}$ is the transmit power per channel and $\eta_{\mathrm{SS}}$ is the nonlinear noise power coefficient, calculated as:

$$
\begin{aligned}
\eta_{\mathrm{SS}}= & \frac{16}{27} \gamma^{2} \frac{B_{\text {ref }}}{R_{s}^{3}} \\
& \cdot \iint_{D}\left|\frac{1-\mathrm{e}^{\left(j 4 \pi^{2}\left|\beta_{2}\right| f_{1} f_{2}-2 \alpha_{\mathrm{np} / \mathrm{km}}\right) L_{s}}}{2 \alpha_{\mathrm{np} / \mathrm{km}}-j 4 \pi^{2}\left|\beta_{2}\right| f_{1} f_{2}}\right|^{2} \mathrm{~d} f_{1} \mathrm{~d} f_{2},
\end{aligned}
$$

where $\gamma$ is the fiber nonlinear coefficient $\left(\mathrm{W}^{-1} \mathrm{~km}^{-1}\right)$ and $\left|\beta_{2}\right|$ is the absolute value of the CD parameter $\left(\mathrm{ps}^{2} \mathrm{~km}^{-1}\right)$. The integration domain $D$ is described in [42] and depends basically on the WDM comb occupation.

In a highly loaded spectrum, incoherent nonlinear noise accumulation [8] can be assumed:

$$
P_{\mathrm{NLI}}=N_{S} P_{\mathrm{NLI}, \mathrm{SS}}=N_{S} \eta_{\mathrm{SS}} P_{\mathrm{Tx}}^{3} .
$$

The OSNR including the linear and nonlinear contributions can thus be written as:

$$
\mathrm{OSNR}=\frac{P_{\mathrm{TX}}}{P_{\mathrm{ASE}}+P_{\mathrm{NLI}}}=\frac{P_{\mathrm{TX}}}{N_{S}\left(P_{\mathrm{ASE}, \mathrm{SS}}+\eta_{\mathrm{SS}} P_{\mathrm{TX}}^{3}\right)},
$$

where $P_{\mathrm{ASE}, \mathrm{SS}}$ is the ASE noise power for a single span.

Therefore, there is an optimum launch power $P_{\mathrm{TX}, \mathrm{OPT}}$ that maximizes the OSNR and, consequently, the transparent reach:

$$
P_{\mathrm{TX}, \mathrm{OPT}}=\left(\frac{P_{\mathrm{ASE}, \mathrm{SS}}}{2 \eta_{\mathrm{SS}}}\right)^{\frac{1}{3}} .
$$

Replacing $P_{\mathrm{TX}, \mathrm{OPT}}$ into (26), gives the maximum OSNR:

$$
\mathrm{OSNR}=\frac{2 P_{\mathrm{TX}, \mathrm{OPT}}}{3 P_{\mathrm{ASE}}} .
$$

Thus, at the optimum transmit power, nonlinear noise power is half of ASE noise power. Now we are in the position to analyze the impact of $R_{S}$ on $P_{\mathrm{NLI}}$ and, consequently, on OSNR (considering both linear and nonlinear effects). According to (24), $\eta_{\mathrm{SS}}$ decreases with the symbol rate, raising the optimum transmit power (27) and, as a consequence, the OSNR (28). Therefore, in the scope of application of the GN model (long links with a highly packed spectrum), increasing the symbol rate may be beneficial to the system in terms of the tolerance to nonlinearities. We illustrate the problem in a case study with $7950-\mathrm{GHz}$-spaced channels, transmitted over $10 \times 100 \mathrm{~km}$ standard single-mode fiber (SSMF) spans, without dispersion compensation. The fiber parameters are as in [45]. The results are shown in Fig. 12, with several OSNR versus transmit power curves for different symbol rates. Clearly, higher symbol rates increase the OSNR at the optimum transmit power.

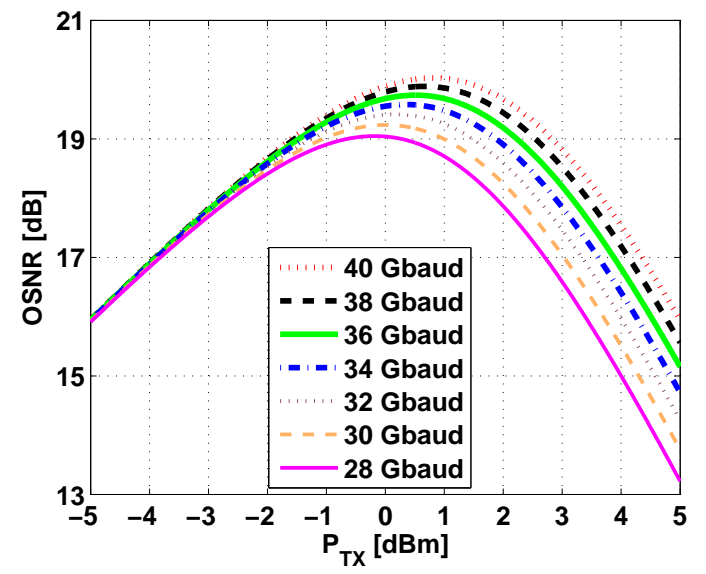

Fig. 12. Achievable OSNR over 10 spans versus transmission power with different symbol rates.

\section{Future Perspectives And Conclusion}

Adaptive optical transceivers, able to sense the channel and take actions, should be a crucial component of future optical networks. The sensing task should be implemented by the chain of signal processing algorithms in the coherent receiver. While polarization-mode dispersion is seamlessly compensated for in the adaptive equalizer, chromatic dispersion requires accurate estimation to set up the static equalizer. A promising method for CD estimation, based on the autocorrelation of the signal power waveform, gives accurate results at low computational complexity.

In future dispersion uncompensated optical links, at the optimum launch power, the amplifier noise power should be twice the nonlinear noise power. Therefore, the ratio between amplifier and nonlinear noise powers is an useful parameter to the system designer. However, in the nonlinear regime, amplifier noise power estimation is still an open research topic and one of the remaining challenges for future adaptive optical transceivers.

The choice of a suitable set of parameters (or an operating point), including modulation format, symbol rate and coding, is also a key task. In relatively short, regeneration-free, optical links, the most suitable operating point maximizes the order of the modulation format (constrained to implementation limitations) and minimizes the symbol rate to reduce the power consumption in the $\mathrm{CD}$ equalizer. Regenerated optical links, in turn, should attempt to reduce the number of regenerators, which are costly and power hungry, by choosing the modulation format, symbol rate and coding schemes that maximize the transparent reach. This is achieved by operating the system at the highest-order modulation format allowed by the technology, and also the highest symbol rate (at low code rates). In some cases, increasing the symbol rate reduces the OSNR required at the receiver and mitigates nonlinearities in dispersion-uncompensated links.

Looking at the future, in unregenerated links the line transmission rate could be adaptively reduced to save power during light traffic periods. Such symbol rate switching is a good example where the transceiver can learn from past 
experience. For example, artificial intelligence techniques can help the transceiver to choose the best time of the day (based on the client data pattern) to trigger the rate switching and avoid unnecessary bouncing. In regenerated optical links, in turn, variable-code-rate transceivers will play a crucial role to extend transparent reach and reduce the number of expensive and power-hungry regenerators. Therefore, FEC adaptation, currently overseen in most studies in the field, should gain momentum as an emerging topic.

\section{ACKNOWLEDGEMENT}

The authors would like to thank the anonymous reviewers for their valuable comments and suggestions to improve the quality of the paper.

\section{REFERENCES}

[1] S. Chandrasekhar and X. Liu, "Terabit superchannels for high spectral efficiency transmission," in Proceedings of ECOC, no. Tu.3.C.5, Sep. 2010.

[2] O. Gerstel, M. Jinno, A. Lord, and S. Yoo, "Elastic optical networking: a new dawn for the optical layer?" IEEE Commun. Mag., vol. 50, no. 2, pp. s12-s20, Feb. 2012.

[3] R. S. Tucker, R. Parthiban, J. Baliga, K. Hinton, R. W. A. Ayre, and W. Sorin, "Evolution of WDM optical IP networks: a cost and energy perspective," J. Lightwave Technol., vol. 27, no. 3, pp. 243-252, Feb. 2009.

[4] W. Wei, C. Wang, and J. Yu, "Cognitive optical networks: key drivers, enabling techniques, and adaptive bandwidth services," IEEE Commun. Mag., vol. 50, no. 1, pp. 106-113, January 2012.

[5] F. Hauske, M. Kuschnerov, B. Spinnler, and B. Lankl, "Optical performance monitoring in digital coherent receivers," J. Lightw. Technol., vol. 27, no. 16, pp. 3623-3631, Aug 2009.

[6] S. Savory, "Digital coherent optical receivers: algorithms and subsystems," IEEE J. Sel. Topics Quantum Electron., vol. 16, no. 5, pp. 11641179, Sept 2010.

[7] R. Borkowski, L. H. H. de Carvalho, E. P. da Silva, J. C. M. Diniz, D. Zibar, J. C. R. de Oliveira, and I. T. Monroy, "Experimental evaluation of prefiltering for 56 Gbaud DP-QPSK signal transmission in $75 \mathrm{GHz}$ WDM grid," Opt. Fiber Technol., vol. 20, no. 1, pp. 39 - 43, 2014.

[8] A. Carena, V. Curri, G. Bosco, P. Poggiolini, and F. Forghieri, "Modeling of the impact of nonlinear propagation effects in uncompensated optical coherent transmission links," J. Lightw. Technol., vol. 30, no. 10, pp. 1524-1539, May 2012.

[9] P. Poggiolini, A. Carena, V. Curri, G. Bosco, and F. Forghieri, "Analytical modeling of nonlinear propagation in uncompensated optical transmission links," IEEE Photon. Technol. Lett., vol. 23, no. 11, pp. $742-744$, Jun. 2011.

[10] Z. Dong, A. P. T. Lau, and C. Lu, "OSNR monitoring for QPSK and 16-QAM systems in presence of fiber nonlinearities for digital coherent receivers," Opt. Express, vol. 20, no. 17, pp. 19520-19534, Aug. 2012.

[11] D. J. Ives, B. C. Thomsen, R. Maher, and S. J. Savory, "Estimating OSNR of equalised QPSK signals," in Proceedings of ECOC, no. Tu.6.A.6, Sep. 2011.

[12] R.-J. Essiambre, G. Kramer, P. Winzer, G. Foschini, and B. Goebel, "Capacity limits of optical fiber networks," J. Lightw. Technol., vol. 28, no. 4, pp. 662-701, Feb. 2010.

[13] M. Cvijetic, Optical Transmission Systems Engineering. Artec House, Inc., 2004.

[14] M. Kuschnerov, F. Hauske, K. Piyawanno, B. Spinnler, A. Napoli, and B. Lankl, "Adaptive chromatic dispersion equalization for non-dispersion managed coherent systems," in Proceedings of $O F C$, no. OMT1, Mar. 2009.

[15] D. Godard, "Self-recovering equalization and carrier tracking in twodimensional data communication systems," IEEE Trans. Commun., vol. 28, no. 11, pp. 1867-1875, 1980.

[16] R. Borkowski, X. Zhang, D. Zibar, R. Younce, and I. T. Monroy, "Experimental adaptive digital performance monitoring for optical DPQPSK coherent receiver,' in Proceedings of ECOC, no. Tu.3.K.5, Sep. 2011.
[17] Q. Sui, A. Lau, and C. Lu, "Fast and robust chromatic dispersion estimation using auto-correlation of signal power waveform for DSP based-coherent systems," in Proceedings of OFC, no. OW4G, Mar. 2012.

[18] F. Pereira, V. Rozental, and D. A. Mello, "Limitations of the power autocorrelation-based chromatic dispersion estimation method in dispersionmanaged links," in Proceedings of LAOP, no. LM4C.4, 2012.

[19] J. Yu, Z. Dong, H.-C. Chien, Y. Shao, and N. Chi, "7-Tb/s $(7 \times 1.284 \mathrm{~Tb} / \mathrm{s} / \mathrm{ch})$ signal transmission over $320 \mathrm{~km}$ using PDM-64QAM modulation," IEEE Photon. Technol. Lett., vol. 24, no. 4, pp. 264-266, Feb. 2012.

[20] J. H. Ke, Y. Gao, and J. Cartledge, "Three-carrier 1 Tbit/s dual polarization 16-QAM superchannel using look-up table correction and optical pulse shaping," in Proceedings of ECOC, no. We.C.1.2, Sep. 2013.

[21] Y.-K. Huang, M.-F. Huang, E. Ip, E. Mateo, P. Ji, D. Qian, A. Tanaka, Y. Shao, T. Wang, Y. Aono, T. Tajima, T. Xia, and G. Wellbrock, "Highcapacity fiber field trial using Terabit/s all-optical OFDM superchannels with DP-QPSK and DP-8QAM/DP-QPSK modulation," J. Lightw. Technol., vol. 31, no. 4, pp. 546-553, Feb. 2013.

[22] M.-F. Huang, A. Tanaka, E. Ip, Y.-K. Huang, D. Qian, Y. Zhang, S. Zhang, P. Ji, I. Djordjevic, T. Wang, Y. Aono, S. Murakami, T. Tajima, T. Xia, and G. Wellbrock, "Terabit/s Nyquist superchannels in high capacity fiber field trials using DP-16QAM and DP-8QAM modulation formats," J. Lightw. Technol., vol. 32, no. 4, pp. 776-782, Feb. 2014.

[23] C. Laperle and M. OSullivan, "Advances in high-speed DACs, ADCs, and DSP for optical coherent transceivers," J. Lightw. Technol., vol. 32, no. 4, pp. 629-643, Feb. 2014.

[24] G. Bosco, V. Curri, A. Carena, P. Poggiolini, and F. Forghieri, "On the performance of Nyquist-WDM terabit superchannels based on PMBPSK, PM-QPSK, PM-8QAM or PM-16QAM subcarriers," J. of Lightw. Technol., vol. 29, no. 1, pp. 53 - 61, Jan. 2011.

[25] A. Morea, S. Spadaro, O. Rival, J. Perello, F. Agraz, and D. Verchere, "Power management of optoelectronic interfaces for dynamic optical networks," in Proceedings of ECOC, no. We.8.K.3, Sep. 2011, pp. 1-3.

[26] F. Chang, K. Onohara, and T. Mizuochi, "Forward error correction for 100 G transport networks," IEEE Commun. Mag., vol. 48, no. 3, pp. S48-S55, Mar. 2010.

[27] C. R. S. Fludger, T. Duthel, D. vanden Borne, C. Schulien, E.-D. Schmidt, T. Wuth, J. Geyer, E. DeMan, G.-D. Khoe, and H. Waardt, "Coherent equalization and POLMUX-RZ-DQPSK for robust 100-GE transmission," J. of Lightw. Technol., vol. 26, no. 1, pp. 64-72, Jan. 2008.

[28] V. N. Rozental, G. Bruno, G. Lombardi, R. Grosso, A. Alping, and D. A. A. Mello, "Cognitive power management in $100 \mathrm{G}$ optical transponders," in Proceedings of ICTON, no. We.D1.3, 2012, pp. 1-4.

[29] W. Freude, R. Schmogrow, B. Nebendahl, D. Hillerkuss, J. Meyer, M. Dreschmann, M. Huebner, J. Becker, C. Koos, and J. Leuthold, "Software-defined optical transmission," in Proceedings of ICTON, no. Tu.D1.1, Jun. 2011

[30] E. Ip, A. P. T. Lau, D. J. F. Barros, and J. M. Kahn, "Coherent detection in optical fiber systems," Opt. Express, vol. 16, no. 2, pp. 753-791, Jan 2008.

[31] S. J. Savory, "Digital filters for coherent optical receivers," Opt. Express, vol. 16 , no. 2 , pp. $804-817,2008$.

[32] A. Faria, L. de Aguiar, D. Lara, and A. Loureiro, "Comparative analysis of power consumption in the implementation of arithmetic algorithms," in Proceedings of Trustcom, Nov. 2011, pp. 1247 -1254.

[33] B. Spinnler, "Equalizer design and complexity for digital coherent receivers," IEEE J. Sel. Topics Quantum Electron., vol. 16, no. 5, pp. 1180 - 1192, Sept.-Oct. 2010.

[34] R. Blahut, Fast Algorithms for Signal Processing. Cambridge University Press, 2010.

[35] J. Proakis and D. Manolakis, Digital signal processing: principles, algorithms, and applications, 3rd ed. Prentice Hall, 1996.

[36] J. C. Geyer, C. R. Fludger, T. Duthel, C. Schulien, and B. Schmauss, "Efficient frequency domain chromatic dispersion compensation in a coherent polmux QPSK-receiver," in Proceedings of OFC, no. OWV5, 2010.

[37] T. M. Cover and J. A. Thomas, Elements of Information Theory, 1st ed. John Wiley \& Sohns, Inc., 1991.

[38] D. Mello, A. Barreto, T. de Lima, T. Portela, L. Beygi, and J. Kahn, "Optical networking with variable-code-rate transceivers," J. Lightw. Technol., vol. 32, no. 2, pp. 257-266, Jan. 2014.

[39] L. Beygi, E. Agrell, J. Kahn, and M. Karlsson, "Rate-adaptive coded modulation for fiber-optic communications," J. Lightw. Technol., vol. 32, no. 2, pp. 333-343, Jan. 2014. 
[40] G.-H. Gho, L. Klak, and J. Kahn, "Rate-adaptive coding for optical fiber transmission systems," J. Lightw. Technol., vol. 29, no. 2, pp. 222-233, Jan. 2011.

[41] M. Arabaci, I. Djordjevic, L. Xu, and T. Wang, "Nonbinary LDPCcoded modulation for rate-adaptive optical fiber communication without bandwidth expansion," IEEE Photon. Technol. Lett., vol. 24, no. 16, pp. 1402-1404, Aug. 2012.

[42] P. Poggiolini, "The GN model of non-linear propagation in uncompensated coherent optical systems," J. Lightw. Technol., vol. 30, no. 24, pp. 3857-3880, Dec. 2012.

[43] R. Dar, M. Feder, A. Mecozzi, , and M. Shtaif, "Properties of nonlinear noise in long, dispersion-uncompensated fiber links," Opt. Express, vol. 21 , no. 22 , p. 2568525699 , Nov. 2013.

[44] A. Carena, G. Bosco, V. Curri, Y. Jiang, P. Poggiolini, and F. Forghieri, "On the accuracy of the GN-Model and on analytical correction terms to improve it," arXiv:1401.6946, Jan. 2014.

[45] G. Bosco, P. Poggiolini, A. Carena, V. Curri, and F. Forghieri, "Analytical results on channel capacity in uncompensated optical links with coherent detection," Opt. Exp., vol. 19, no. 26, pp. 440-451, Dec. 2011.

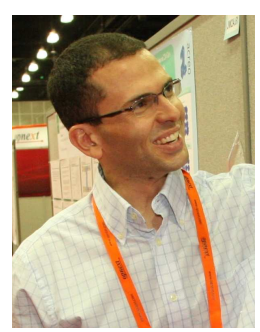

Darli A. A. Mello studied electrical engineering at the RWTH-Aachen, Aachen, Germany, and at the State University of Campinas (Unicamp), Campinas, Brazil, where he graduated in 2000. He received the M.Sc. degree from the Munich University of Technology (TUM), Munich, Germany, in 2002. During his Master's studies, he carried out both experimental and theoretical work at the Siemens research labs in Munich. He received the Ph.D. degree from Unicamp in 2006. After his Ph.D. studies, he joined Padtec as a Senior Technology Engineer. There, he was responsible for R\&D project coordination, system conception and design, and for cooperative projects with universities. From 2008 to 2013 he was an Assistant Professor at the University of Brasilia, Brasilia, Brazil. Since 2014 he has been with the School of Electrical and Computer Engineering (FEEC), Unicamp, as assistant professor. His main research interests are optical transmission and networking. Dr. Mello was the TPC Co-Chair of the 30th Brazilian Telecommunications Symposium, 2012, and is part of subcommittee N3 of OFC.

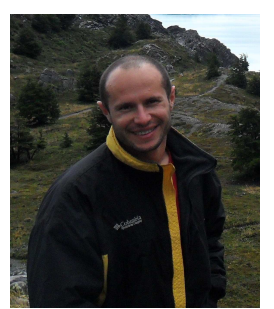

Valery Nobl Rozental studied Electrical Engineering at the University of Brasilia, where he obtained his B. Sc. and M. Sc. degrees in 2009 and 2011, respectively. During his Master's studies, Valery spent several months working for Ericsson at the Optical Communication Lab in Backnang, Germany. In 2012-2013 he worked with the Ericsson Optical Communication R\&D Group in Genoa, Italy. Valery is currently a $\mathrm{PhD}$ student at the University of Brasilia. In 2014 he joined the Telecommunications Research and Development Center (CPqD), Campinas, Brazil. His research area is the long-haul, ultra-high-speed optical transmission, involving advanced modulation formats, software-defined and cognitive optical transmitters and digital signal processing.

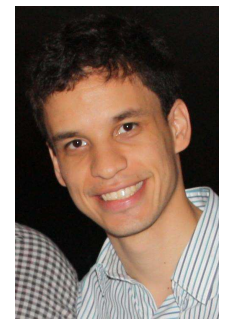

Tiago C. Lima has a degree in Electrical Engineering from the University of Brasilia, Brasilia, Brazil, in 2012, where he is currently working towards the Master's degree. He was a trainee at Globo TV and spent six months as an exchange student at the University of Coimbra, Coimbra, Portugal. His research interests are in error-correcting codes, fiber nonlinear effects and link adaptation for optical networks.

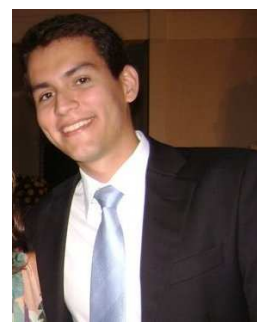

Fernando Cota obtained his B. Sc. degree in Communication Networks Engineering from University of Brasilia, Brazil, in 2011. In the same year, he joined the OCNLab, where he worked on Ericssons project involving superchannels. He is currently a Master's student at the University of Brasilia and his research area is estimation of optical channel conditions.

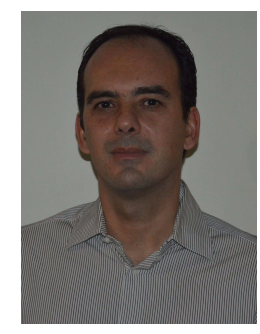

André Noll Barreto (Dr. Ing. - TU Dresden, 2001) graduated in Electrical Engineering at the Catholic University (PUC) in Rio de Janeiro, Brazil in 1994, where he also earned a Master's degree in 1996. In 2001 he obtained his Dr.Ing. with highest honours (summa cum laude) at the Dresden University of Technology, Germany. He is since 2009 assistant professor at the University of Braslia (UnB), Brazil. $\mathrm{He}$ also held positions previously at the IBM Research Center in Zurich, Switzerland, at Claro, in Rio de Janeiro and at Nokia Institute of Technology (INdT), in Braslia. He is the author of four international patents and numerous articles in journals and in international conferences. His current areas of interest are green wireless networks, broadband wireless communication systems PHY and MAC, spectrum sensing for cognitive radio, and transmission techniques for advanced optical communication systems

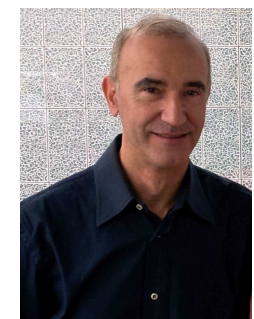

Marco Camera has expertise in both electrical and optical transmission. He started his experience as a designer of line terminals and regenerators from $2 \mathrm{Mb}$ to $8 \mathrm{Mb}$, then continued with optical front-ends for plesiochronous and synchronous interfaces from $34 \mathrm{Mb}$ to $40 \mathrm{~Gb}$. In the last 10 years he followed research activities on ultra-high-speed transmission (40Gb-100Gb and beyond) acquiring strong knowledge of optical performances and solutions needed to satisfy the transport requirements of photonic systems. With his current role of Ericsson's senior expert, he is a reference in Optical Networks Development and Strategic Sourcing. He leads the collaboration with universities at the Ericsson Optical Research Center, promoting national and international projects.

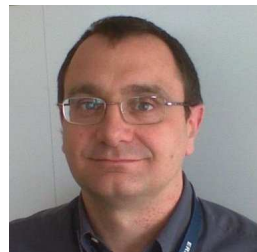

Gianmarco Bruno is a member of Systems and Technology group in the Development Unit Optical and Metro at Ericsson, Genova (Italy). He has been working with Ericsson (formerly Marconi plc) since 2000 in the field of optical transport systems development. His research interests are in the field of WDM optical transmission, network modeling and optimization. He is author of several publications and patents in the field of optical networking. Since 2007 Gianmarco is active in the ITU-T SG15 question dealing with optical physical layer standardization and is an IEEE member. He received the Dr. Ing. degree in Electronic Engineering cum laude from the University of Genoa, Italy, in 1999. 